\title{
DERIVATIVE FORMULAS FOR BESSEL, STRUVE AND ANGER-WEBER FUNCTIONS
}

\author{
RoBERT E. GAUNT
}

Abstract. We derive formulas for the derivatives of general order for the functions $z^{-v} h_{v}(z)$ and $z^{v} h_{v}(z)$, where $h_{v}(z)$ is a Bessel, Struve or Anger-Weber function.

Mathematics subject classification (2010): Primary 26A24, 33C10, Secondary 33C99. Keywords and phrases: Differentiation, Bessel functions, Struve functions, Anger-Weber functions.

\section{REFERENCES}

[1] R. E. Gaunt, Rates of Convergence of Variance-Gamma Approximations via Stein's Method, DPhil thesis, University of Oxford, 2013.

[2] R. E. GaUnt, Variance-Gamma approximation via Stein's method, Electron. J. Probab., 19, no. 38 (2014), 1-33.

[3] N. Nielsen, Handbuch der Theorie der Cylinderfunktionen, Leipzig, 1904.

[4] F. W. J. Olver, D. W. Lozier, R. F. Boisvert AND C. W. Clark, NIST Handbook of Mathematical Functions, Cambridge University Press, 2010.

[5] G. N. Watson, A Treatise on the Theory of Bessel Functions, 2nd ed. Cambridge, England: Cambridge University Press, 1966.

[6] G. N. Watson, On Nielsen's functional equations, Messenger, XLVIII. (1919), 49-53. 